\title{
Let Us Do Not Forget Durability
}

\author{
John Somberg
}

There have been considerable advances in cardiovascular device-based therapeutics. We have seen several waves of drug alluding stents, biodegradable stents, closure devices and percutaneous valve implants and valve repair devices. These innovations have made great strides with marked patient benefits in the short run. Being able to avoid open heart surgery offers our patient's major quality of life benefits. Being able to avoid coronary artery bypass surgery, open heart surgery for valve repair, or replacement has been very favorably received by patients. Closure of a foramen ovalie, an atrial or ventricle septal defect has opened new vistas in interventional cardiology.

Cardiovascular research has focused on the benefits of these percutaneous procedures, their early success and early complication rates. What is just as critical, but far harder to determine is the long-term outcomes of these procedures. What is the durability of the procedure and how does it compare to the more invasive procedures? These comparisons are critical for physicians and patients to decide on which approach to choose. We have a device approval process that focuses on the initial success of a device and its risks and benefits. Unfortunately, we focus less on long-term durability. If we have longterm follow-up, we have at most 1 or 2 years of follow-up. For a clinical trial this is indeed a long follow-up, but for a patient who has a device base procedure, long term may be measured in one, two, or three decades not years.

We need to ask for follow-up evaluations that may last a decade or longer. We cannot require this long a follow-up before regulatory approval. That would deny patients beneficial therapy for years and destroy innovation in these areas of cardiovascular research. However, we need careful long-term follow-up to assess device and procedure durability. This is different from depending upon case reports being published or voluntary adverse reporting by physicians. Systematic long-term follow-up of new devices and procedures is critical. When nothing could be done for aortic stenosis, the short-term benefits of any valve replacement were acceptable. Now that we have both invasive open-heart procedures along with transarterial procedures, research on the long-term duality needs to be undertaken to inform evidence-based decisions. Registries following the continued success rate of devices and adverse events can make an important contribution. Perhaps a review of approval decisions every decade and how they compare to other alternative, more established approaches is something Food and Drug Administration (FDA) and National Heart, Lung, and Blood Institute (NHLBI) should undertake for the public good. Physicians and investigators cannot readily address these issues on their own. A national policy evaluating long-term durability is long overdue.

\section{Acknowledgments}

None to declare.

\section{Financial Disclosure}

None to declare.

\section{Conflict of Interest}

None to declare.

\section{Data Availability}

The author declare that data supporting the findings of this study are available within the article. 